\title{
Assessing the contribution of active and passive stresses in C. elegans elongation
}

\author{
Martine Ben Amar, ${ }^{1,2, *}$ Paul Qiuyang-Qu, ${ }^{1}$ Thanh Thi Kim Vuong-Brender, ${ }^{3}$ and Michel Labouesse ${ }^{4}$ \\ ${ }^{1}$ Laboratoire de Physique Statistique, Ecole Normale Supérieure,
PSL Research University; Sorbonne Universités UPMC Unv Paris 06; CNRS; 24 rue Lhomond, 75005 Paris, France \\ ${ }^{2}$ Institut Universitaire de Cancérologie, Faculté de médecine, \\ Université Pierre et Marie Curie-Paris 6, 91 Bd de l'Hôpital, 75013 Paris, France \\ ${ }^{3}$ MRC Laboratory of Molecular Biology, Francis Crick Avenue, \\ Cambridge Biomedical Campus, Cambridge CB2 OQH, United Kingdom \\ ${ }^{4}$ Laboratoire de Biologie du Développement - Institut de Biologie Paris Seine (LBD - IBPS), \\ Sorbonne Universit'es, UPMC Univ Paris 06, CNRS, Paris, France
}

\begin{abstract}
The role of the actomyosin network is investigated in the elongation of $C$. elegans during embryonic morphogenesis. We present a model of active elongating matter that combines pre-stress and passive stress in non-linear elasticity. Using this model we revisit recently published data from laser ablation experiments to account for why cells under contraction can lead to an opening fracture. By taking into account the specific embryo geometry, we obtain quantitative predictions for the contractile forces exerted by the molecular motors myosin II for an elongation up to $70 \%$ of the initial length. This study demonstrates the importance of active processes in embryonic morphogenesis and the interplay between geometry and nonlinear mechanics during morphological events. In particular, it outlines the role of each connected layer of the epidermis compressed by an apical extra-cellular matrix that distributes the stresses during elongation.
\end{abstract}

Mechanical stresses play a crucial role in animal embryogenesis. At the macroscopic level, differential growth generates compressive stresses creating the circumvolutions of intestine [1-3], brain cortex [4-6] and fingerprints of skin [7, 8]. The folding of tissues is then directly linked to the coupling between volumetric growth, tissue properties and geometry. At the cellular level, the interplay between mechanics and morphological events such as division, migration and tissue organization is much more subtle. The high deformability of cells is counter-balanced by the cellular filament networks, especially by the actomyosin cortex. It comprises a network of cross-linked actin filaments located below the plasma membrane, so that the local cell contractility results from the myosin molecular motors which transform the chemical energy of ATP hydrolysis into contractile stresses. How these microscopic processes at the cell level cooperate to induce shape transition at the tissue level is central in "active matter". At early stages in small organisms such as Drosophila [9] or C. elegans [10], the number of cells is relatively small and the structure is simple enough, giving perhaps a way to bridge scales between microscopic activity and observable tissue displacements. Both of these biological species are considered as model systems where the theoretical framework of active matter [11-15] can be applied and more importantly quantified by analyzing experimental data. Here we investigate the early stage of $C$. elegans elongation up to $70 \%$ when the embryo contains 65 epidermal cells in cortical position in an ovoid shell. Among available approaches, laser cuts in different locations on alive embryos allow to evaluate either the tension or the stiffness [16], quantities necessary to assess the active stresses at the origin of the elongation [17, 18]. Since in this process, there is no cytokinesis or apoptosis and no position exchange between neighboring cells, we select a continuum approach of active matter to predict the fracture opening. However, as highlighted in $[19,20]$, the difficulty in active matter consists in evaluating the stresses: active or passive for samples with complex geometry and elastic properties. To this end, one must comprehend the nonlinearities of shape transformations in these small organisms with a limited information on mechanical quantities.

The aim of this letter is then to investigate this problematics when only some characteristics are known for C. elegans embryos. Our scope is to estimate the activity of the molecular motors and to compare it to the elastic resistance. For that, we establish an analytical model based on nonlinear elasticity for soft tissues and compare our predictions with measurements by laser ablation [16]. The proposed treatment can be adapted to other morphogenetic events in embryogenesis.

Laser ablation.-Fracture opening gives a way to determine the forces at the cellular level. This technique helps to experimentally deduce the tensile stress in the perpendicular direction to the fracture line when the cell stiffness is known. Conversely, when the stresses are well identified, it gives some insights on the fiber network organization. Indeed, the crack opening in linear elasticity is an ellipse [21] and the shape factor (opening $b_{i}$ along $x_{i}$ divided by the crack length $l_{k}$ along $x_{k}$ ) is given by: $\mathcal{F}_{i k}=b_{i} / l_{k} \sim 2 \sigma_{i} / E$ where $\sigma_{i}$ is the tension and $E$ the Young modulus, see FIG.(1c). For $\mathcal{F}_{i k}$, other stress components than $\sigma_{i}$ do not play a role and for an anisotropic sample, $E$ must be replaced by $E_{i}$ the stiffness in the $x_{i}$ direction [16].

In a recent work [16], Vuong-Brender et al. apply this technique in different parts of C. elegans (in FIG.(1d)) and demonstrate that the cracks always open both in the dorsoventral (DV) and in the anterior-posterior (AP) directions. If the crack opening is not a surprise in AP, it is more puzzling 
(a)

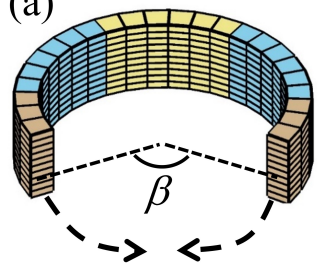

(c)

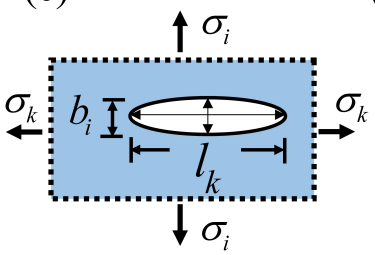

(b)

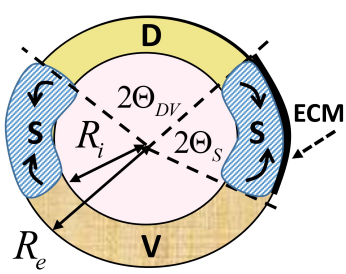

(d)

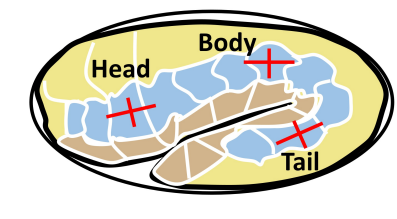

FIG. 1: The ventral enclosure of C. elegans. (a) Schematic representation before enclosure; (b) Horizontal section at enclosure. Different colors are chosen: yellow for the dorsal (D), blue for the seam (S) and brown for the ventral (V) cells. (c) Schema of a planar fracture under tensile stresses. (d) Position of laser fractures achieved in the embryo [16].

in the DV direction for various reasons FIG.(1d). First, cracks cannot open in compression (except in some very specific conditions [22]). Because of volume conservation, an extension in AP leads automatically to a contraction in DV. In addition, Myosin II, the actin molecular motors have been observed [23-26] in the seam (S) domain (blue in FIG.(1a, b, d)), and these motors are contractile. Even more intriguing, in $\mathrm{S}$ cells, the opening is larger in the DV than in the AP direction [16]. The theoretical interpretation of these experiments cannot be captured by the linear elasticity framework. To recover the shape-factor $\mathcal{F}_{i k}$ requires evaluating first the state of stresses or strains inside the embryo before elongation and then incorporating the active stresses due to molecular motors. By coupling them, the modeling must recover the results of the laser ablation but also the possibility to elongate the embryo up to $70 \%$. Nonetheless, in nonlinear elasticity, and to the best of our knowledge, there is no general formula for crack opening but only local analysis of the stresses at both ends [27, 28]. A simple analogy between linear/nonlinear elasticities suggests to replace $\sigma$ by the equivalent Cauchy stress $\sigma_{i}$ and $E$ by the local stiffness $E_{i}$. The following addresses the evaluation of these two quantities in the nonlinear elasticity framework beginning first by the geometry analysis.

Geometry and strains.-The morphogenetic events of the embryo elongation consist of cell intercalation and ventral enclosure [10]. The displacements of matter are strongly constrained by the limited space and induce significant strains [29]. In particular, the ventral enclosure, schematized by FIG.(1a), cannot be achieved without local forces to join the two parts of the epithelial cortex, as demonstrated by myosin

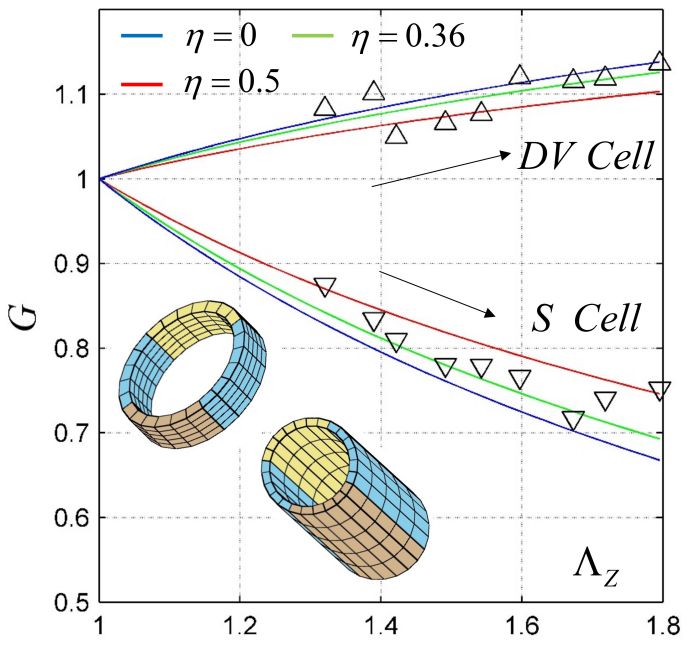

FIG. 2: Angular stretch for $\mathrm{S}$ and DV cells. Notice that $G_{S}<1$ for $\mathrm{S}$ and $G_{D V}>1$ for D cells. Theoretical curves, explained in [30] are weakly dependent of the epidermis thickness, represented by $\eta=1-\left(R_{i} / R_{e}\right)^{2}$. Comparison with experimental data from [16].

accumulation [23, 24]. Hence, when elongation begins, the tissues have already stored pre-strains and pre-stresses [20] and the measurements in [16] result from those cumulated stresses. Their evaluation requires a complete knowledge of the history, which is difficult to assess. A possible simplified scenario will be the enclosure of a cylindrical partial shell (with a lacking angular sector $\beta$, see FIG.(1a) by ortho-radial stretching. But this shell is made of a non-homogeneous epidermis with 3 kinds of cells called hereafter dorsal (D), seam (S) and ventral (V) cells, see FIG.(1b). Once the suture is achieved, the embryo becomes a composite cylinder made of a row of epithelial cells and nascent intestine. Besides, it is covered by the extra-cellular matrix (ECM), a thin layer of secreted proteins. In the stress-free configuration, before enclosure, the D cells occupy one sector between $\left[-\phi_{D V}, \phi_{D V}\right]$, $\mathrm{S}$ cells are located between $\left[\phi_{D V}, \tilde{\beta}-\phi_{D V}\right]$ and $\mathrm{V}$ cells fill the remaining sector up to $\tilde{\beta}=\pi-\beta / 2$ ( FIG.(1a) and in [30]). From the mechanical viewpoint, we do not make distinction between the $\mathrm{D}$ and $\mathrm{V}$ cells and now call them DV cells. As shown in [30], at full enclosure (FE, FIG.(1b)), the position of material points are defined by $R, \Theta, Z$ and then becomes $r, \theta, z$ with elongation. At $\mathbf{F E}$, the epidermis lies between the inner $R_{i}$ and the outer $R_{e}$ radius. It is possible to map the stress-free configuration onto the current one and then to define the elastic strains, but one must keep in mind that the experimental results refer to the beginning of elongation which is not a stress-free state. This distinction is essential in nonlinear elasticity. We call $G$ the angular stretch defined by $\theta=G \Theta_{i}$, which varies with the axial stretch $\Lambda_{Z}$ and the domain area. We hypothesize that at FE, all parts are stretched in the ortho-radial direction so $G_{0}>1$. The two unknown 
parameters of the initial geometry (e.g. $\tilde{\beta}$ and $\Theta_{D V}$ ) are determined in [30] by arclength measurements [16]. Looking for the simplest solution where strains and stresses remain diagonal, the deformation gradient tensor defined by $\mathbf{F}=$ Diag $\left(\Lambda_{R}, \Lambda, \Lambda_{Z}\right)$ is then $\mathbf{F}=\operatorname{Diag}(\partial r / \partial R, G r / R, d z / d Z)$. Resulting from both enclosure and elongation, the elastic tensor $\mathbf{F}_{\mathbf{e}}$ defined by $\operatorname{Diag}\left(\lambda_{R}, \lambda, \lambda_{Z}\right)$ reads $\mathbf{F}_{\mathbf{e}}=\mathbf{F} \mathbf{F}_{\mathbf{0}} . \mathbf{F}_{\mathbf{0}}$ is the pre-stretch tensor defined by 2 independent eigenvalues: $\mathbf{F}_{\mathbf{0}}=\operatorname{Diag}\left(\left(\lambda_{0} \lambda_{0 Z}\right)^{-1}, \lambda_{0}, \lambda_{0 Z}\right)$, since the incompressibility imposes $\lambda_{R}=1 /\left(\lambda \lambda_{Z}\right)$. (See also [30]). These tensors are defined everywhere in the cylinder and are different in $\mathrm{S}$ or DV domains. Only the elongations $\Lambda_{Z}$ and $\lambda_{0 Z}$ will remain identical in all parts for reason of integrity. Since there is no cell division during the process, the volume conservation in the epithelium gives:

$$
r^{2}-r_{i}^{2}=\frac{1}{G \Lambda_{Z}}\left(R^{2}-R_{i}^{2}\right)
$$

which allows to calculate $G$, knowing that at the border of the interior zone, $\Lambda=r_{i} / R_{i}=1 / \sqrt{\Lambda_{Z}}$. In [30], it is shown how the angular stretch $G$ for S and DV cells, FIG.(2), are obtained from measurements of the circumferential lengths published in [16]. Since data are available only at $\Lambda_{Z}=1.3,1.5$, extrapolation at $\Lambda_{Z}=1$ is used. Agreement between modeling and experimental data [30] validates the first steps with an epithelium thickness of order $2 \mu \mathrm{m}$. Mechanical stresses can now be evaluated.

Equilibrium equations and boundary conditions.-The crack opening reaches its finite value after only few seconds, which is in the same order of magnitude as the velocities of actomyosin flows (in the order of $1 \mu \mathrm{m} / \mathrm{s}$. [31, 32]). Focussing on the equilibrium value of the slit opening, we can neglect viscoelasticity $[33,34]$. Then, in cylindrical geometry, the Cauchy stress $\sigma$, diagonal as the deformation gradient tensor $\mathbf{F}_{\mathrm{e}}$, satisfies:

$$
\frac{\partial \sigma_{r}}{\partial r}+\frac{1}{r}\left(\sigma_{r}-\sigma_{\theta}\right)=0
$$

where $\sigma_{r}$ and $\sigma_{\theta}$ are the radial and ortho-radial components in the current configuration. This equation is identical in linear elasticity [35]. Eq.(2) requires only one boundary condition, chosen at the apical border, just below the ECM whose thickness is about $10^{-2}$ the embryo radius $[16,26]$. So, it imposes a weak compressive surface stress, $\sigma_{r} \sim 0$, during elongation. Defining $W_{P}$ as the passive elastic energy density, each stress component becomes $\sigma_{k}=\lambda_{k}\left(\partial W_{P} / \partial \lambda_{k}\right)+\sigma_{k}^{a}-p$ $[17,18]$, where $p$ is a Lagrange parameter ensuring the incompressibility and $\sigma_{k}^{a}$ the active stress which only exists in the $\mathrm{S}$ cells. $\sigma_{k}^{a}$ can be decomposed into a volumetric $\sigma^{a, v}$ and a deviatoric $\boldsymbol{\sigma}^{a, d}$ part defined by: $\boldsymbol{\sigma}^{a, v}=\zeta_{a, v} \mathbf{I}$ and $\boldsymbol{\sigma}^{a, d}=\zeta$ $\operatorname{Diag}(0,1,-1)[14,19]$. $\zeta_{a, v}$ may be included into the Lagrange parameter $p$ (a detailed demonstration can be found in [30]). Conversely, the deviatoric part is a traceless tensor with no specific sign. Finally, the definition of a new energy functional [36]: $\tilde{W}_{P}=W_{P}\left(\left(\lambda \lambda_{Z}\right)^{-1}, \lambda, \lambda_{Z}\right)$ enforces auto- matically incompressibility giving:

$$
\left\{\begin{array}{l}
\sigma_{\theta}=\sigma_{\theta}^{p}+\sigma_{r}+\zeta ; \sigma_{z}=\sigma_{Z}^{p}+\sigma_{r}-\zeta \\
\sigma_{\theta}^{p}=\lambda \frac{\partial \tilde{W}_{P}}{\partial \lambda} ; \sigma_{Z}^{p}=\lambda_{Z} \frac{\partial \tilde{W}_{P}}{\partial \lambda_{Z}}
\end{array}\right.
$$

where $\sigma_{\theta}^{p}$ and $\sigma_{Z}^{p}$ decouple from the active part $\zeta$. Once Eq.(2) is solved, all stresses can be calculated explicitly, [30]. Since fractures are made superficially on the outer surface where $\sigma_{r} \sim 0$, only $\sigma_{\theta}$ and $\sigma_{z}$ are the components of interest for our study.

Evaluation of the stresses and fracture opening. - In these epithelial cells, it was found [25] that both micro-tubules and actin filaments are oriented mainly in the ortho-radial direction in both cells. So we choose the simplest constitutive law as a superposition of a matrix and a fiber network elasticity:

$$
W_{P}=\frac{\mu}{2}\left(\lambda_{R}^{2}+\lambda^{2}+\lambda_{Z}^{2}-3\right)+\frac{\tau}{4}\left(\lambda^{2}-1\right)^{2}
$$

when orientation along $\theta$ is imposed. Such superposition is currently achieved with some variants concerning the last term $[37,38]$. Choosing the $\mathrm{S}$ cell coefficient $\mu_{S}$ as unit of elastic energy, we estimate that $\mu_{D V}>1$ to represent a stiffer material and $\tau_{S}<\tau_{D V}$, to represent a weaker degree of fiber alignment in $\mathrm{S}$ cells. Since orientation is the same for actin or micro-tubules [16, 25], a unique coefficient $\tau$ involves both filaments.

At FE, the state of the cylinder is characterized by 3 independent pre-strain quantities which are the ortho-radial stretches $\left(\lambda_{0 S}\right.$ and $\left.\lambda_{0 D V}\right)$ and the axial stretch $\lambda_{0 Z}$, see [30]. Within the thin epithelium approximation, $\eta=1-$ $R_{i}^{2} / R^{2}<<1$, one easily finds $G_{0} \sim \lambda_{0} \sqrt{\lambda_{0 Z}}$, from Eq.(1). From the geometry, the lacking angle of the sector reads $\beta \sim 2(\pi-\tilde{\beta}) \sim 2 \pi\left(1-\left(\sqrt{\lambda_{0 Z}} \lambda_{0 S}\right)^{-1}\right)$. In addition, $\lambda_{0 S}$ and $\lambda_{0 D V}$ values must be compatible with the continuity of the ortho-radial stresses $\sigma_{\theta, S}=\sigma_{\theta, D V}$ at $\mathbf{F E}$ which fixes the ratio of stiffnesses between $\mathrm{S}$ and DV cells with $\lambda_{0 D V}<\lambda_{0 S}$. Post enclosure, the pre-strains modify the elastic strains into: $\lambda=\lambda_{0} \Lambda=\lambda_{0} G r / R$ and $\lambda_{Z}=\lambda_{0 Z} \Lambda_{Z}$ (see [30]). This explains why, a tissue remains in tension even if contractile motors exert a compressive work on it. For a thin epithelium, the elastic stretch $\lambda$ is transformed into $\lambda \sim \lambda_{0} G / \sqrt{\Lambda_{Z}}$ and we have now $\sigma_{\theta, S}^{p}+\zeta=\sigma_{\theta, D V}^{p}$ which gives $\zeta$. $G_{S}$ decreases as $\Lambda_{Z}$ increases leading to a decrease of the passive stress $\sigma_{\theta}^{p}$. However the active stress $\zeta$, an increasing function of $\Lambda_{Z}$, compensates the opening of cracks in the $D V$ direction. Finally, because the epithelial elasticity is both orthotropic and nonlinear, the adapted mathematical formula for the shapefactor $\mathcal{F}_{i k}$ is deduced from $W_{P}$, Eq.(4). The equivalent Young modulus in the $i^{\text {th }}$ direction reads:

$$
E_{i}=K_{i i}-\frac{K_{i j}^{2}}{K_{j j}} ; K_{i j}=\lambda_{j} \frac{\partial \sigma_{i}^{p}}{\partial \lambda_{j}} \text { and } \mathcal{F} \sim 2 \frac{\sigma_{i}^{p}+\zeta_{i}}{E_{i}}
$$

This evaluation presents no difficulty once the elastic energy density is known, albeit this question remains challenging for small organisms.

Results and discussion.-The incompressibility hypothesis and the cylindrical shape are tested by comparing the angular 
(a) Crack opening in Seam cells

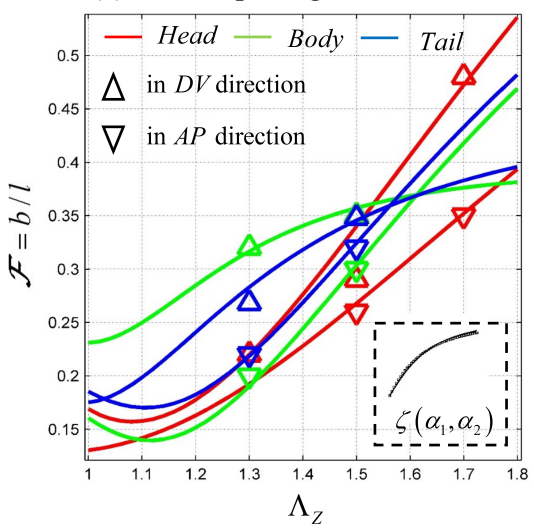

(b) Stress evolution in Seam cells

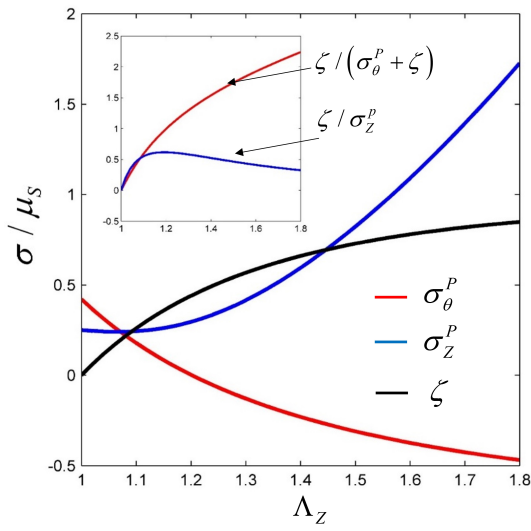

(c) Active stress during elongation

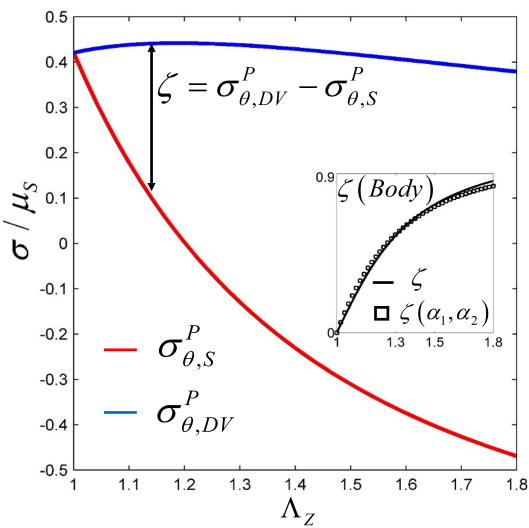

FIG. 3: (a) Crack opening in S cells for head, body and tail in DV or AP directions, Eq.(5). Difference between curves comes from pre-strain values, Table I and [30]. In the insert, the active stress $\zeta$ beginning at enclosure. (b) Comparison of passive versus active stress in $\mathrm{S}$ cells (Eqs. $(3,4)$. The scale for stresses is the stiffness of S cells $\mu_{s}=1$. (c) Active stress evaluated as the difference of $\sigma_{\theta}^{p}$ between DV and S cells. In the insert, $\zeta$, deduced from the model and approximated by $\zeta=2 \alpha_{1} \pi^{-1} \tan ^{-1} \alpha_{2}\left(\Lambda_{Z}-1\right)$ with 2 parameters given in Table I.

stretch $G$ with the experimental values of each domain: seam, dorsal and ventral (see FIG.(2) and Table I). There is a slow dependence with the thickness of the epidermis which is reassuring since this thickness in the order of $2 \mu \mathrm{m}$ is not known with precision. By extrapolation, we derive a value of each arclength at the "supposed" beginning of elongation and finally an angle of order $\beta \sim 26^{\circ}$, indicating a significant pre-stretch at enclosure, associated to a pre-stress about 0.45 for $\sigma_{\theta}$ and 0.35 for $\sigma_{Z}$. As shown in FIG.(3)(b,c), the amplitude of the active stress $\zeta$ is an increasing function of $\Lambda_{Z}$ which saturates around the value 1.8. Above this value, a new mechanism involving muscle cells [39-41] occurs, not considered here as we focus on the role of the actomyosin network. Once the elastic energy $W_{P}$ is obtained from the body results, this energy function is fixed everywhere: in the head and in the tail, only the pre-stretch values due to enclosure are very slightly modified as shown in Table I. After, the theoretical curves are derived from $\mathcal{F}$, Eq. (5) and shown in FIG(3a). Notice that the active stress $\zeta$ is derived from the difference between passive parts of S and DV cells with an empirical formula of 2 parameters: $\zeta=2 \alpha_{1} \pi^{-1} \tan ^{-1} \alpha_{2}\left(\Lambda_{Z}-1\right)$. The agreement is good for the crack opening in $\mathrm{S}$ cells. All the results concerning this elongation step in the $C$. elegans embryonic life are gathered in Table I. The methodology to derive these parameters which rest on the border conditions and available experimental data is explained in details in [30].

To conclude, as emphasized in [20], it is especially delicate to extract quantitative informations from nonlinear mechanical systems involving active and passive stresses and in addition pre-stretch and pre-stress. The complexity increases with the geometry for a multi-layered inhomogeneous epidermis trapped between a central intestine and the apical ECM. In vivo measurements, difficult at the scale of the cell are made possible thanks to the technique of laser ablation which, com- bined with this analysis, gives a satisfactory picture of how molecular motors can achieve cell and embryo deformations. Even if the focus is put on C. elegans geometry, the theory developed here can be adapted to other systems where laser ablation is achieved to assess stresses and illustrates how pre-stress can be accounted for in vivo. The role of mechanics in embryogenesis needs not be demonstrated anymore. However, it is crucial to develop experimental and theoretical tools to fully understand the origin of morphogenetic events in model systems.

Table I:.Geometric and elastic parameters of the model in different parts of the embryo:

\begin{tabular}{c|cc|ccc|cc}
\hline \hline & $\begin{array}{c}C_{0 S} \\
\mu \mathrm{m}\end{array}$ & $\begin{array}{c}C_{0 D V} \\
\mu \mathrm{m}\end{array}$ & $\lambda_{0 Z}$ & $\lambda_{0 S}$ & $\lambda_{0 D V}$ & $\alpha_{1}$ & $\alpha_{2}$ \\
& 14.5 & 33.0 & 1.025 & 1.06 & 1.0326 & 2.2 & 1.27 \\
Head & 14 & & & & \\
Body & 10.1 & 24.8 & 1.025 & 1.09 & 1.05 & 1.15 & 3.2 \\
Tail & 10.1 & 24.8 & 1.055 & 1.05 & 1.0232 & 1.25 & 2.9 \\
\hline
\end{tabular}

Elastic coefficients: $\mu_{D V}=1.44, \tau_{S}=0.15, \tau_{D V}=0.67$

The circumference lengths $C_{0}$ are extrapolated from [16, 30]. $\lambda_{0}$, values at enclosure, differ in $\mathrm{S}$ and DV and from head to tail. The coefficients $\alpha$ 's refer to active stress evolution, see FIG.(3c). The elastic parameters of Eq.(4) do not vary along the embryo.

MBA would like to thank the Isaac Newton Institute of Mathematical Sciences, Cambridge, for support and hospitality during the programme "Growth, Form and SelfOrganization" where work on this paper was undertaken. MBA acknowledges the partial support from the Simons Foundation, the EPSRC grant no EP/K032208/1 and from ANR under the contract EPIMORPH (ANR-18-CE13-0008). PQQ acknowledges the support of the China Scholarship Council (CSC), file $N^{0} 201706100182$. ML thanks the European Research Council (grant 294744) for support. 
Correspondingauthor:M.BenAmar:E-mail: benamarelps.ens. fr

[1] A. J. Coulombre and J. L. Coulombre. Intestinal development: I. morphogenesis of the villi and musculature. Development, 6(3):403-411, 1958.

[2] E. Hannezo, J. Prost, and J.-F. Joanny. Instabilities of monolayered epithelia: shape and structure of villi and crypts. Phys. Rev. Lett., 107(7):078104, 2011.

[3] M. Ben Amar and F. Jia. Anisotropic growth shapes intestinal tissues during embryogenesis. Proc. Natl. Acad. Sci. U.S.A., 110(26):10525-10530, 2013.

[4] R. Toro and Y. Burnod. A morphogenetic model for the development of cortical convolutions. Cereb. Cortex, 15(12):19001913, 2005.

[5] A. Goriely, M. G. Geers, G. A. Holzapfel, J. Jayamohan, A. Jérusalem, S. Sivaloganathan, W. Squier, J. A. van Dommelen, S. Waters, and E. Kuhl. Mechanics of the brain: perspectives, challenges, and opportunities. Biomech. Model. Mechanobiol., 14(5):931-965, 2015.

[6] M. Ben Amar and A. Bordner. Mimicking cortex convolutions through the wrinkling of growing soft bilayers. J. Elast., 129(12):213-238, 2017.

[7] M. Kücken and A. C. Newell. Fingerprint formation. J. Theor. Biol., 235(1):71-83, 2005.

[8] P. Ciarletta and M. Ben Amar. Papillary networks in the dermal-epidermal junction of skin: a biomechanical model. Mech. Res. Commun., 42:68-76, 2012.

[9] I. Heemskerk, T. Lecuit, and L. LeGoff. Dynamic clonal analysis based on chronic in vivo imaging allows multiscale quantification of growth in the drosophila wing disc. Development, 141(11):2339-2348, 2014.

[10] A. Chisholm and J. Hardin. Epidermal morphogenesis, wormbook, ed. the C. elegans research community. WormBook, 2005.

[11] P. Nardinocchi and L. Teresi. On the active response of soft living tissues. J. Elast., 88(1):27-39, 2007.

[12] F. Jülicher, K. Kruse, J. Prost, and J.-F. Joanny. Active behavior of the cytoskeleton. Phys. Rep., 449(1-3):3-28, 2007.

[13] M. C. Marchetti, J.-F. Joanny, S. Ramaswamy, T. B. Liverpool, J. Prost, M. Rao, and R. A. Simha. Hydrodynamics of soft active matter. Rev. Mod. Phys., 85(3):1143, 2013.

[14] J. Prost, F. Jülicher, and J.-F. Joanny. Active gel physics. Nat. Phys., 11(2):111, 2015.

[15] S. Redemann, J. Baumgart, N. Lindow, M. Shelley, E. Nazockdast, A. Kratz, S. Prohaska, J. Brugués, S. Fürthauer, and T. Müller-Reichert. C. elegans chromosomes connect to centrosomes by anchoring into the spindle network. Nat. Commun., 8:15288, 2017.

[16] T. T. K. Vuong-Brender, M. Ben Amar, J. Pontabry, and M. Labouesse. The interplay of stiffness and force anisotropies drives embryo elongation. Elife, 6:e23866, 2017.

[17] A. Goriely. Five ways to model active processes in elastic solids: Active forces, active stresses, active strains, active fibers, and active metrics. Mech. Res. Commun., 2017.

[18] F. Jülicher, S. W. Grill, and G. Salbreux. Hydrodynamic theory of active matter. Rep. Prog. Phys., 81(7):076601, 2018.

[19] E. Fischer-Friedrich, Y. Toyoda, C. J. Cattin, D. J. Müller, A. A. Hyman, and F. Jülicher. Rheology of the active cell cortex in mitosis. Biophys. J., 111(3):589-600, 2016.

[20] E. Fischer-Friedrich. Active prestress leads to an apparent stiffening of cells through geometrical effects. Biophys. J., 114(2):419-424, 2018.
[21] P. Theocaris, D. Pazis, and B. Konstantellos. The exact shape of a deformed internal slant crack under biaxial loading. Int. J. Fract., 30(2):135-153, 1986.

[22] A. Lucantonio, G. Noselli, X. Trepat, A. DeSimone, and M. Arroyo. Hydraulic fracture and toughening of a brittle layer bonded to a hydrogel. Phys. Rev. Lett., 115(18):188105, 2015.

[23] C. Gally, F. Wissler, H. Zahreddine, S. Quintin, F. Landmann, and M. Labouesse. Myosin ii regulation during C. elegans embryonic elongation: Let-502/rock, mrck-1 and pak-1, three kinases with different roles. Development, 136(18):3109-3119, 2009.

[24] N. Fotopoulos, D. Wernike, Y. Chen, N. Makil, A. Marte, and A. Piekny. Caenorhabditis elegans anillin (ani-1) regulates neuroblast cytokinesis and epidermal morphogenesis during embryonic development. Dev. Biol., 383(1):61-74, 2013.

[25] S. Quintin, S. Wang, J. Pontabry, A. Bender, F. Robin, V. Hyenne, F. Landmann, C. Gally, K. Oegema, and M. Labouesse. Non-centrosomal epidermal microtubules act in parallel to let-502/rock to promote C. elegans elongation. Development, 143(1):160-173, 2016.

[26] T. T. K. Vuong-Brender, S. K. Suman, and M. Labouesse. The apical ecm preserves embryonic integrity and distributes mechanical stress during morphogenesis. Development, pages 144(23):4336-4349, 2017.

[27] Z. Suo. Singularities, interfaces and cracks in dissimilar anisotropic media. Proc. Royal Soc. Lond. A, 427(1873):331358, 1990 .

[28] M. Ben Amar. Creases and cracks in finite elasticity. Mech. Res. Commun., in press 2018.

[29] P. Ciarletta, M. Ben Amar and M. Labouesse. Continuum model of epithelial morphogenesis during caenorhabditis elegans embryonic elongation. Philos. Trans. Royal Soc. A, 367(1902):3379-3400, 2009.

[30] See the supplement.

[31] A.-C. Reymann, F. Staniscia, A. Erzberger, G. Salbreux, and S. W. Grill. Cortical flow aligns actin filaments to form a furrow. Elife, 5:e17807, 2016.

[32] A. Saha, M. Nishikawa, M. Behrndt, C.-P. Heisenberg, F. Jülicher, and S. W. Grill. Determining physical properties of the cell cortex. Biophys. J., 110(6):1421-1429, 2016.

[33] I. Bonnet, P. Marcq, F. Bosveld, L. Fetler, Y. Bellache and F. Graner Mechanical state, material properties and continuous description of an epithelial tissue J. R. Soc. Interface 9 26142623 (2012); DOI: 10.1098/rsif.2012.0263.

[34] S. Tlili, E. Gauquelin, B. Li, O. Cardoso, B. Ladoux, H. Delano-Ayari, F. Graner Collective cell migration without proliferation: density determines cell velocity and wave velocity preprint

[35] L. D. Landau and E. M. Lifshitz. Course of theoretical physics. Elsevier, 2013.

[36] R. W. Ogden. Non-linear elastic deformations. Courier Corporation, 1997.

[37] T. C. Gasser, R. W. Ogden, and G. A. Holzapfel. Hyperelastic modelling of arterial layers with distributed collagen fibre orientations. J. Royal Soc. Interface, 3(6):15-35, 2006.

[38] G. A. Holzapfel, J. A. Niestrawska, R. W. Ogden, A. J. Reinisch, and A. J. Schriefl. Modelling non-symmetric collagen fibre dispersion in arterial walls. J. Royal Soc. Interface, 12(106):20150188, 2015.

[39] B. D. Williams and R. H. Waterston. Genes critical for muscle development and function in caenorhabditis elegans identified through lethal mutations. J. Cell Biol., 124(4):475-490, 1994.

[40] H. Zhang, F. Landmann, H. Zahreddine, D. Rodriguez, M. Koch, and M. Labouesse. A tension-induced mechanotrans- 
duction pathway promotes epithelial morphogenesis. Nature, 471(7336):99, 2011.

[41] Rafaela De Vita, R. Grange, Paola Nardinocchi, and Luciano
Teresi. Mathematical model for isometric and isotonic muscle contractions. Journ. Theoretical Biology, 425:1-10, 2017. 


\title{
Assessing the contribution of active and passive stresses in C. elegans elongation
}

\author{
Martine Ben Amar, ${ }^{1,2, *}$ Paul Qiuyang-Qu, ${ }^{1}$ Thanh Thi Kim Vuong-Brender, ${ }^{3}$ and Michel Labouesse ${ }^{4}$ \\ ILaboratoire de Physique Statistique, Ecole Normale Supérieure,
PSL Research University; Sorbonne Universités UPMC Univ Paris 06; CNRS; 24 rue Lhomond, 75005 Paris, France \\ ${ }^{2}$ Institut Universitaire de Cancérologie, Faculté de médecine, \\ Université Pierre et Marie Curie-Paris 6, 91 Bd de l'Hôpital, 75013 Paris, France \\ ${ }^{3}$ MRC Laboratory of Molecular Biology, Francis Crick Avenue, \\ Cambridge Biomedical Campus, Cambridge CB2 OQH, United Kingdom \\ ${ }^{4}$ Laboratoire de Biologie du Développement - Institut de Biologie Paris Seine (LBD - IBPS), \\ Sorbonne Universit'es, UPMC Univ Paris 06, CNRS, Paris, France
}

\section{DETERMINATION OF THE COMPOSITE GEOMETRY AND DEFORMATIONS}

\section{Geometry}

Our model assumes 2 steps for the elongation process: The first one, that we call BE, before enclosure, is the transformation of the embryo from a cylinder not fully surrounded by an epithelium (configuration IE) into a closed one (configuration FE), as shown in FIG. (1a) and FIG. (1b) of the main manuscript or here in the schema (1). The second is the elongation process PE just after the full enclosure (FE). Even if we maintain the cylindrical geometry from $\mathbf{B E}$ to $\mathbf{P E}$, we should take care of the change of topology. At IE, the embryo geometry is defined by 2 angles $\phi_{D V}$ and $\phi_{S}$, giving the missing angle $\beta$ :

$$
2\left(\phi_{D V}+\phi_{S}\right)=\pi-\beta / 2
$$

Calling $\Theta$ the new angular position of each point inside the epithelium at $\mathbf{F E}$, the angular stretch $G_{0}$ and the sector angles which mark out the DV from the $\mathrm{S}$ domain read:

$$
G_{0}=\Theta / \phi \quad \text { and } \quad \Theta_{D V}+\Theta_{S}=G_{0, D V} \phi_{D V}+G_{0, S} \phi_{S}=\pi / 2
$$

From now on, we define the observable position of a material point by capital letters $(R, \Theta, Z)$ which is transformed into small letters $(r, \theta, z)$ as the elongation proceeds (during PE). With this definition, an homogeneous elongation from FE is given by $\Lambda_{Z}=z / Z$. The composite structure of the sample leads to a change of angular position in each domain giving an angular stretch $G=\theta / \Theta$ with the constraint: $\theta_{D V}+\theta_{S} \equiv \pi / 2$.

\section{Definition of the geometric stretches}

Applying the incompressibility property to each embryo component (DV or S), we obtain:

$$
r^{2}-r_{i}^{2}=\frac{1}{G \Lambda_{Z}}\left(R^{2}-R_{i}^{2}\right)
$$

$R_{i}$ (resp. $r_{i}$ ) is the position of the inner boundary between the epithelium and the inner zone at FE (resp. during PE). In the inner part, Eq. (3) reduces to $r=R / \sqrt{\Lambda_{Z}}$ so the continuity for $r=r_{i}$ at the border imposes : $r_{i}=R_{i} / \sqrt{\Lambda_{Z}}$. In addition, assuming that everywhere the same axial extension leads to a unique value of the stretch $\Lambda_{Z}$ in all parts of the cylinder.

$$
r^{2}=\frac{R_{i}^{2}}{\Lambda_{Z}}+\frac{1}{G \Lambda_{Z}}\left(R^{2}-R_{i}^{2}\right)
$$

Consequently, at this stage we have defined the observable deformation quantities and the gradient deformation tensor $\mathbf{F}=\operatorname{Diag}\left(\Lambda_{R}, \Lambda, \Lambda_{Z}\right)$ in function of 3 stretches:

$$
\Lambda_{R}=\frac{\partial r}{\partial R}, \quad \Lambda=\frac{r G}{R} \text { and } \Lambda_{Z}
$$




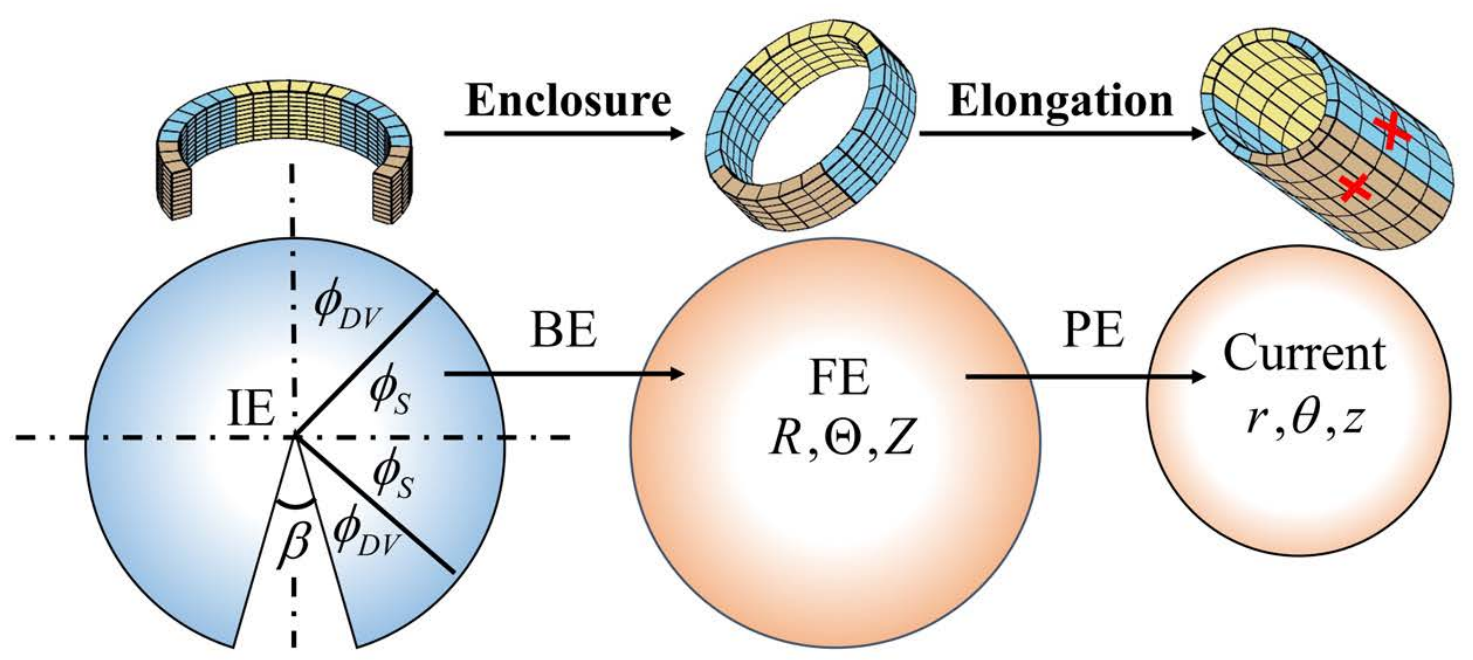

FIG. 1: Schematic representation of the 2 steps involved in the model and the 3 configurations: initial, at enclosure and current. Notice the red crosses after elongation corresponding to the laser cuts.
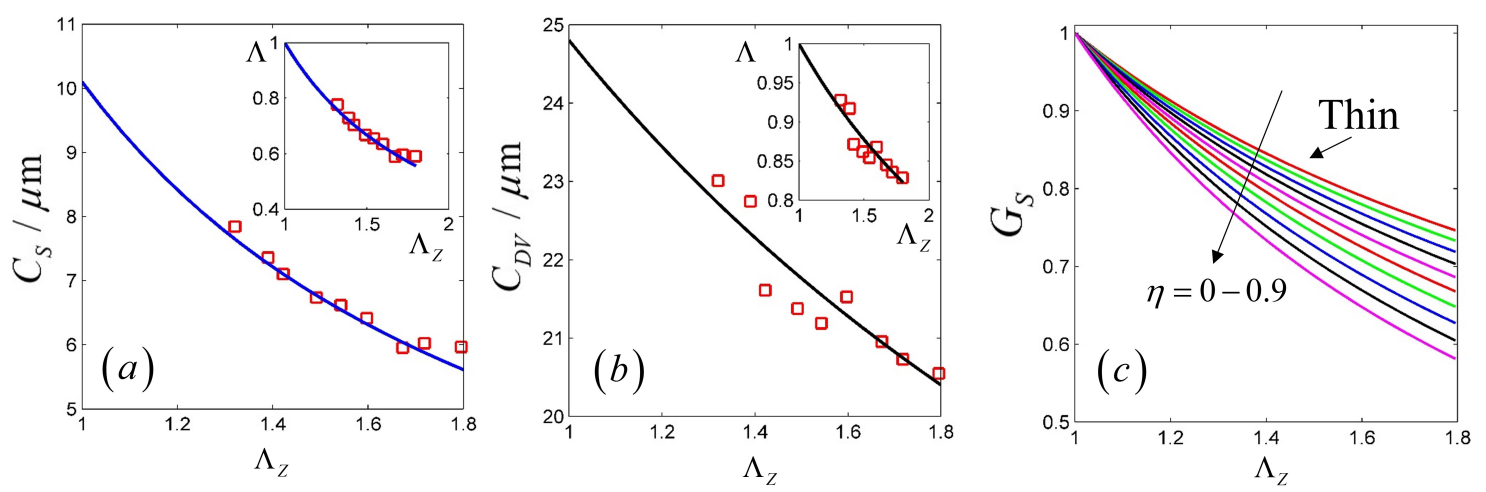

FIG. 2: (a) Experimental circumferential length of the seam cellular domain extracted from [1]. In blue the best fitting curve corresponding to $C=C_{0} \Lambda$ and the ortho-radial stretch in the inset of top. (b) Same results for the DV cells analytically deduced using Eq.(6) and Eq.(7). Experimental points from [1] are included for comparison. (c) Theoretical $G_{S}$ value as a function of $\Lambda_{Z}$ according to Eq.(7) when the epithelium thickness is varied. For DV cells, see Figure (2) of the main text.

As $\Lambda_{Z}$ increases, the circumferential length $C$ of each cellular domain has been measured [1] and is directly related to $\Lambda$ since $C=C_{0} \Lambda$, with $C_{0}$ the value at $\mathbf{F E}$. In addition, we have the following geometric constraints:

$$
C_{0, D V} \Lambda_{D V}+C_{0, S} \Lambda_{S}=\frac{C_{0, S}+C_{0, D V}}{\sqrt{\Lambda_{Z}}}
$$

Before any elastic evaluation, one needs to know the size of the components of the sample under investigation. Unfortunately at this scale, not all of them are known with precision. Focussing on circumferential length shown in FIG. (2), we extract both the best fitting function $C_{S}\left(\Lambda_{Z}\right)$ during $\mathbf{P E}$ and also the initial value $C_{0, S}$ at FE. After we can easily get $\Lambda_{S}=C_{S} / C_{0, S}$. Now one needs also the $G$ value as a function of $\Lambda_{Z}$. With Eqs. (3), (4) and (5), the angular extension $G_{S}$ is derived by inversion of $\Lambda$ according to:

$$
\Lambda=\frac{G}{\sqrt{\Lambda_{Z}}} \sqrt{1+\left(G^{-1}-1\right) \eta} \text { leading to } G=\frac{-\eta+\sqrt{\eta^{2}+4(1-\eta) \Lambda^{2} \Lambda_{Z}}}{2(1-\eta)}
$$

where $\eta=1-R_{i}^{2} / R^{2}$ is a biologic data, function of the ratio between the inner and the outer radius of the epithelium. On the surface of the epithelia we take $\Lambda=\Lambda\left(R=R_{e}\right)$ with $\eta=1-R_{i}^{2} / R_{e}^{2}$, approximatively 2 times the ratio of the epithelium thickness versus the radius of the embryo at enclosure. So having $\Lambda$, one can extract $G_{S}$ from Eq.(7) if the thickness of the epithelium is known. An estimate of about $2 \mu \mathrm{m}\left(R_{e}-R_{i} \approx 2 \mu \mathrm{m}\right.$ and $\left.R_{e} \approx 10 \mu \mathrm{m}\right)$ has been chosen for an evaluation of $G_{S}$ 
compared with experimental data points. We do the same for $D V$ after an evaluation of $C_{0, D V}$, but here we use the geometrical constraint Eq.(6) to deduce $\Lambda_{D V}$ and $G_{D V}$. Once the theoretical $G_{D V}$ is determined, we include the experimental data for comparison. The good agreement validates the cylindrical approach for a thin epithelium.

Finally, as elongation proceeds, an angular contraction is shown for the $\mathrm{S}$ cells (i.e. $G_{S}<1$ ) and an extension for the $D V$ domain (i.e. $G_{D V}>1$ ) responsible for the whole extension of the embryo (see FIG. (2) of the main text). With the above analysis, the cylindric composite picture of the embryo is coherent. More specifically we can draw the conclusion that, after enclosure, the geometric ortho-radial stretch is tensile for DV cells but contractile for seam cells (without consideration of the active stress and pre-stretch). In other words, we expect that cracks in the DV cells always open, in the seam they open in the AP direction but should close in the DV direction. This is not confirmed by the experiment.

\section{STRAIN VERSUS STRESS AND THE FRACTURE OPENING}

Once the geometric strains are acquired, the elastic strains $\lambda, \lambda_{R}$, and $\lambda_{Z}$ which minimize the elastic energy density are then:

$$
\lambda_{Z}=\Lambda_{Z} \lambda_{0 Z}, \lambda=\Lambda \lambda_{0} \text { and } \lambda_{R}=\left(\lambda \lambda_{Z}\right)^{-1}
$$

where $\lambda_{0}$ means the stretch prior to observation, dependent on $G_{0}$.

\section{Elastic energy models}

The elastic energy density for living tissues is a function of the stretches but there exists several models especially when fibers play a role. Here, we choose the simplest one, superposition of a matrix term represented by the usual Neo-Hookean energy contribution (or Flory energy) and a "fiber term" which affects the elasticity in the direction of the fibers. Since it is difficult to stretch fibers or filaments, an increase of the elastic energy is expected in the direction of stretching. Such anisotropy has been treated by an orthotropic linear model in [1]. In S or DV cells, assuming that the orientation of the fibers are mostly in the ortho-radial direction, we have:

$$
\left\{\begin{array}{l}
W_{S}=\frac{\mu_{S}}{2}\left(\lambda_{R}^{2}+\lambda^{2}+\lambda_{Z}^{2}-3\right)+\frac{\tau_{S}}{4}\left(\lambda^{2}-1\right)^{2} \\
W_{D V}=\frac{\mu_{D V}}{2}\left(\lambda_{R}^{2}+\lambda^{2}+\lambda_{Z}^{2}-3\right)+\frac{\tau_{D V}}{4}\left(\lambda^{2}-1\right)^{2}
\end{array}\right.
$$

where the stretches $\lambda_{i}$ refer to Eq.(8). In the following, we will choose the coefficient $\mu_{S}$ as stress unit, so it will be fixed to 1 . In the theory of non-linear elasticity, the first term in $W_{S}$ and $W_{D}$ is called the first invariant and the second one the forth invariant. This representation is standard and can be found in text books [2-4]. In partially disordered fiber networks, the stiffness coefficient $\tau_{i}$ defined in Eq. (9) decreases for the benefit of the first coefficient $\mu_{i} . \tau_{S}$ is expected to be less than $\tau_{D V}$ since the fibers in the seam cells are less ordered than in the DV cells. In our macroscopic representation, due to the ortho-radial direction of the fiber networks, we do not need to distinguish microtubules from actin filaments. At this stage 3 coefficients are unknown, however the ortho-radial stress must be continuous at the border between $\mathrm{S}$ and $\mathrm{D}$ domains.

\section{The stresses}

The Cauchy stress is the superposition of passive and active stresses $\boldsymbol{\sigma}=\boldsymbol{\sigma}^{p}+\boldsymbol{\sigma}^{a} . \boldsymbol{\sigma}^{a}$ may be decomposed into an isotropic and a deviatoric tensors:

$$
\boldsymbol{\sigma}^{a}=\zeta_{a, v} \operatorname{Diag}(1,1,1)+\zeta \operatorname{Diag}(0,1,-1)
$$

where the deviatoric part takes into account the orientation of the actin filaments. The elastic or passive part reads:

$$
\sigma_{i}^{p}=\lambda_{i} \frac{\partial W}{\partial \lambda_{i}}-p
$$

$p$ being the Lagrange parameter which allows to impose the incompressibility condition, that is: $\lambda_{R} \cdot \lambda \cdot \lambda_{Z}=1$. This condition can be introduced in Eq. (9) to eliminate $\lambda_{R}$ which plays a minor role during elongation, in this system. Restricting on the space 
of incompressible deformation states, we can define a new energy density $\tilde{W}_{P}\left(\lambda, \lambda_{Z}\right)=W_{P}\left(\left(\lambda \lambda_{Z}\right)^{-1}, \lambda, \lambda_{Z}\right)$. Derivatives of $\tilde{W}_{P}$ with respect to the elastic stretch $\lambda$ and $\lambda_{Z}$ give:

$$
\frac{\partial \tilde{W}}{\partial \lambda}=-\frac{1}{\lambda^{2} \lambda_{Z}} \frac{\partial W}{\partial \lambda_{R}}+\frac{\partial W}{\partial \lambda}
$$

and

$$
\frac{\partial \tilde{W}}{\partial \lambda_{Z}}=-\frac{1}{\lambda \lambda_{Z}^{2}} \frac{\partial W}{\partial \lambda_{R}}+\frac{\partial W}{\partial \lambda_{Z}}
$$

Finally one gets for the total Cauchy stress, superposition of active and passive parts:

$$
\left\{\begin{array}{l}
\sigma_{r}=\lambda_{R} \frac{\partial W}{\partial \lambda_{R}}-p+\zeta_{a, v} \\
\sigma_{\theta}=\lambda \frac{\partial \tilde{W}}{\partial \lambda}+\lambda_{R} \frac{\partial W}{\partial \lambda_{R}}-p+\zeta_{a, v}+\zeta \\
\sigma_{z}=\lambda_{Z} \frac{\partial \tilde{W}}{\partial \lambda_{z}}+\lambda_{R} \frac{\partial W}{\partial \lambda_{R}}-p+\zeta_{a, v}-\zeta
\end{array}\right.
$$

where the two last equations take into account Eqs. (12) and (13). Finally, it reads:

$$
\left\{\begin{array}{lll}
\sigma_{\theta}=\sigma_{\theta}^{p}+\sigma_{r}+\zeta & \text { where } & \sigma_{\theta}^{p}=\lambda \frac{\partial \tilde{W}}{\partial \lambda} \\
\sigma_{z}=\sigma_{z}^{p}+\sigma_{r}-\zeta & \text { where } & \sigma_{z}^{p}=\lambda_{Z} \frac{\partial \tilde{W}}{\partial \lambda_{z}}
\end{array}\right.
$$

Notice that, in the main manuscript, $\sigma_{r} \sim 0$ since the laser cuts are made superficially.

\section{Fracture opening}

In the work of Theocaris et al. [5], the slit opening is deduced from linear elasticity. In [1], the method is extended to anisotropic elasticity and to the incremental treatment of nonlinear elastic modeling. Here we modify the Theocaris result replacing the Hookean stress by the Cauchy stress and the Young modulus by its local evaluation. These quantities are derived from the energy density Eq. (13).

$$
\mathcal{F}_{i k}=\frac{b_{i}}{l_{k}} \sim 2 \frac{\sigma_{i}}{E} \quad \Longrightarrow \quad \mathcal{F} \sim 2 \frac{\sigma_{i}^{p}+\zeta_{i}}{E_{i}}
$$

With

$$
E_{i}=K_{i i}-\frac{K_{i j}^{2}}{K_{j j}} \text { where } K_{i j}=\lambda_{j} \frac{\partial \sigma_{i}^{P}}{\partial \lambda_{j}} \text { and } \mathcal{F} \sim 2 \frac{\sigma_{i}^{p}+\zeta_{i}}{E_{i}}
$$

\section{BOUNDARY CONDITIONS AND CONSTRAINTS ON PARAMETERS}

\section{Continuity of the ortho-radial stress}

The ortho-radial stress must be continuous between the S and DV cells: For S cells, we have:

$$
\sigma_{\theta, S}=\lambda_{S}^{2}\left(1-\frac{1}{\lambda_{S}^{4} \lambda_{S Z}^{2}}\right)+\tau_{S} \lambda_{S}^{2}\left(\lambda_{S}^{2}-1\right)+\zeta\left(\Lambda_{Z}\right)
$$

For DV cells:

$$
\sigma_{\theta, D V}=\mu_{D V} \lambda_{D V}^{2}\left(1-\frac{1}{\lambda_{D V}^{4} \lambda_{D V, Z}^{2}}\right)+\tau_{D V} \lambda_{D V}^{2}\left(\lambda_{D V}^{2}-1\right)
$$



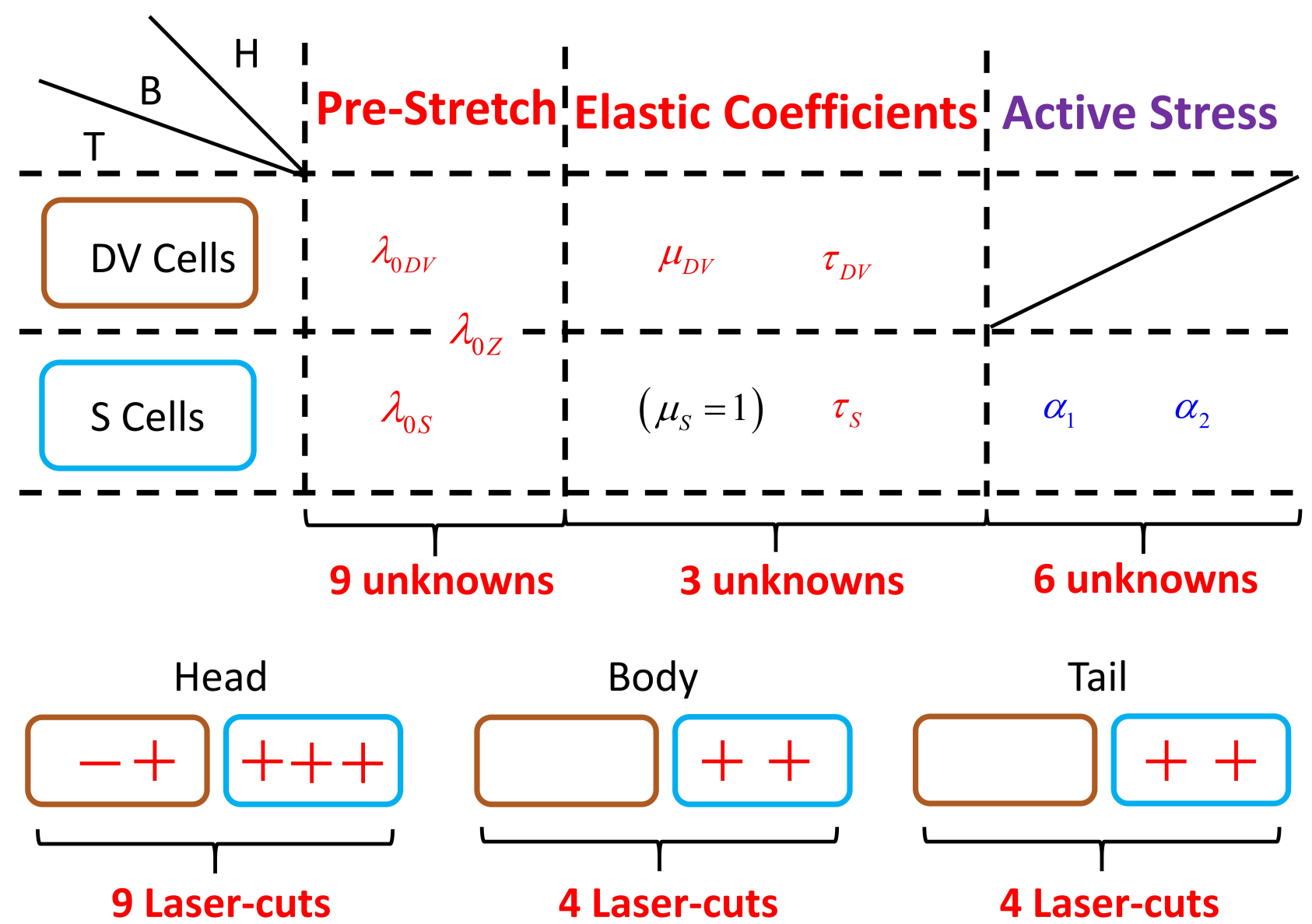

FIG. 3: Table of parameters and available data from [1]. It concerns 3 positions in the embryo: head, body and tail and 2 different kinds of cells $\mathrm{S}$ and DV. Number of unknowns: 18, measurements: 17 and continuity equations: 10.

At the junction between $\mathrm{S}$ and DV, the mechanical equilibrium imposes:

$$
\sigma_{\theta, S}=\sigma_{\theta, D V}
$$

Finally the number of modeling parameters is 18 if we consider that 2 parameters describe the active stress: 3 pre-stretch values per position, 3 parameters for the density energy and 2 for the active stress in each position. The number of available measurements concerning crack opening is 17 . But we also have constraints coming from the balance of ortho-radial stress $\left(\sigma_{S, \theta}=\sigma_{D V, \theta}\right)$ at the DV-S border : 3 values of $\Lambda_{Z}=0,1.3,1.5$ and 4 for the head, which gives 10 constraints on coefficients. So our treatment is not pure fitting since the number of unknowns is less than available data. We can confirm that we successfully explain the fracture opening and in addition to that, the determination of tissue characteristics is correct.

\section{METHODOLOGY FOR EVALUATION OF PARAMETERS}

With a large number of unknowns, the procedure for nonlinear fitting employed here is iterative. In a first step, we fix all pre-stretch values to 1 and focus on the elastic coefficients $\mu_{i}$ and $\tau_{i}$ of the body cells. Then the number of unknowns (5) is determined by the data of fracture (4) and the constraint of continuity of the stress for $\Lambda_{Z}=1$. It gives an initial estimate shared by head, body and tail. Fortunately, once the stiffness is given, the other parameters decouple in each part respectively. We solve them separately. Having a good estimate for pre-stretch values imposed to 1, we approach the total 18 parameters from 17 experimental data and 3 stress continuity equations (see Eq. (20). We relax the other continuity equations introducing them into the sum of the error functions to minimize iteratively. 
* Correspondingauthor:M.BenAmar:E-mail:benamarelps.ens.fr

[1] T. T. K. Vuong-Brender, M. Ben Amar, J. Pontabry, and M. Labouesse. The interplay of stiffness and force anisotropies drives embryo elongation. Elife, 6:e23866, 2017.

[2] Ogden R.W. Non-linear elastic deformations Dover Publications (1997) and Ellis Horwood (1984)

[3] G.A. Holzapfel Nonlinear Solid Mechanics ,Wiley (2010)

[4] A. Goriely, The Mathematics and Mechanics of Biological Growth.Interdisciplinary Applied Mathematics, Springer (2017)

[5] P. Theocaris, D. Pazis, and B. Konstantellos. The exact shape of a deformed internal slant crack under biaxial loading. Int. J. Fract., 30(2):135-153, 1986 